\title{
Prediction of mycotoxin risks due to climate change in Korea
}

\author{
Ho-Sung Lee ${ }^{1}$ Nam ji Kwon' ${ }^{2}$ Yongsoo Kim³ $\cdot$ Hunjoo Lee ${ }^{2}$
}

Received: 15 January 2018/Accepted: 23 April 2018/Published online: 14 May 2018

(C) The Korean Society for Applied Biological Chemistry 2018

\begin{abstract}
Climate change has been considered as a main threat for food safety by influencing on crop production and food supply chain through the change in temperature and humidity. To prevent risks of mycotoxins from climate change, it is important to predict mycotoxin risks with statistical approaches and stepwise process to compile large volume of datasets, such as climate change, contamination level, and cultivation area in specific regions. This paper aims at prioritization of vulnerable mycotoxins related to climate change in Korea. In addition, this paper focuses on prioritization of vulnerable raw materials for specific mycotoxins and prediction of vulnerable regions for vulnerable raw materials in Korea. Among six target mycotoxins (deoxynivalenol, fumonisin (B1 and B2), ochratoxin A, patulin, total aflatoxin (B1, B2, G1, and G2), and zearalenone), ochratoxin A (OTA) and total aflatoxin (TA) were identified as specific vulnerable mycotoxins. In addition, 4 raw materials (chestnut, dried red pepper, perilla seed, and soy bean) were identified as vulnerable raw materials for OTA and TA and vulnerable regions were predicted to be moved to the northward areas in Korea. These results can be utilized to design long-term national sampling plan for mycotoxins in food considering climate change in Korea.
\end{abstract}

Hunjoo Lee

adstar@cheminet.kr

1 Department of Biomedical Engineering, Kyunghee University, Yongin-si, South Korea

2 CHEM.I.NET Ltd., Room 302, 773-3 Mok-dong, Yangcheon-gu, Seoul, South Korea

3 Korea Health Industry Development Institute, 187 Osongsaengmyeong2(i)-ro, Gangoe-myeon, Cheongwon-gun, Chungcheongbuk-do 363-951, South Korea
Keywords Climate change $\cdot$ Food safety $\cdot$ Mycotoxin Ochratoxin A - Total aflatoxin

\section{Introduction}

Climate change has been considered as a main threat for food safety by influencing on crop production and food supply chain through the change in temperature and humidity [1]. The favorable change in growth conditions such as temperature and water activity for mycotoxigenic fungi has possibility to increase mycotoxin production [2]. A public health perspective on mycotoxins can cause disease or death in human and animals that consumed foods infected by mycotoxins due to their toxic and carcinogenic characteristics [1-5], which could be limited in specific mycotoxins and regions vulnerable to climate change [5]. Therefore, it is important to predict how climate change will affect mycotoxins in food [5] and to establish spatialtemporal risk management policies for mycotoxins because the pace or pattern of climate change will vary depending on the region or the country.

Until now, many studies have been continuously conducted to predict mycotoxin risks due to climate change around the world [2-4, 6-15]. For example, one study [4] predicted a decrease in coffee production influenced by increased risks of mycotoxins such as ochratoxin A (OTA), fumonisins, and aflatoxins as a result of climate change. This study also suggested risk management strategies that it would be important to conduct good agricultural practices and prepare post-harvest processes including proper techniques for raw coffee to decrease the level of OTA contamination in coffee [4]. 
To prevent future threats from mycotoxin risks due to climate change, prediction models can be used as preemptive and strategic risk management tools for food production and safety. For development of highly reliable prediction models, it is crucial to secure sufficient data including (1) long-term past weather data and predicted climate data based on future scenarios, (2) contaminationlevel data for mycotoxins in food monitored by national representative sampling, and (3) cultivation area data describing the exposure intensity related as it relates to climate in Korea.

Although required datasets were acquired, it is very difficult to organize and compile those datasets systemically because each dataset was generated by the different governmental authorities with each different file format, respectively.

Therefore, there is a need to (1) conduct data processing and cleaning such as standardization and mapping of heterogeneous code sets for data linkage and (2) integrate datasets between climate change data and other sources. Due to the large volume of datasets such as climate data, statistical analysis and modeling processes are required to predict mycotoxin risks due to climate change.

This study focused on (1) preliminary collection and compilation of required datasets, (2) prioritization of specific mycotoxins related to climate change in Korea, (3) prioritization of vulnerable raw materials for specific mycotoxins, and (4) prediction of vulnerable regions for vulnerable raw materials progressively. This study described the stepwise processes for prediction of risks due to climate change in Kora and presented its results with figures.

\section{Materials and methods}

\section{Preliminary collection and compilation of required datasets}

As precedent process, four datasets-(1) climate change, (2) cultivation area, (3) recipes, and (4) mycotoxin concentrations for processed foods-were collected for prediction of the mycotoxin risk preliminary. These datasets were produced by Korean governmental authorities (Korea Meteorological Administration; KMA [16], Korea AgroFisheries \& Food Trade Corp./Food Information Statistics System; AT/FIS [17], Ministry of food and drug safety; MFDS [18, 19], Ministry of Agriculture, Food and Rural Affairs; MAFRA [20]). Table 1 shows information including data description, total number of records, and references for each dataset.

As shown in Table 1, climate change datasets ( $n=2,947,251)$ were collected from KMA, including past observation data $(n=486,807)$ from 1999 to 2015 and future projected data $(n=2,460,444)$ based on the Representative Concentration Pathways (RCP) scenario presented by the 5 th report of the Intergovernmental Panel on Climate Change [5] between 2015 and 2100. The RCP scenario had four pathways: RCP 2.6, 4.5, 6.0, and 8.5. In RCP 2.6, an extreme reduction policy was implemented, but it was impossible to realize, while RCP 4.5 enforced reduction policies considerably. Additionally, RCP 6.0 carried out reduction policies at an appropriate level and RCP 8.5 equated to the present status, which had no implemented reduction policy [6]. Datasets for cultivation areas by crops $(n=267,133)$ were collected from the Web site of AT/FIS. Datasets for recipes of processed foods $(n=758)$ collected from MFDS included recipe data for each food (e.g., bread and soy sauce). Datasets for mycotoxin concentrations $(n=67,292)$ included those of processed foods $(n=61,229)$ and raw materials $(n=6,063)$. Data for processed foods were collected from MFDS which monitored six mycotoxins-deoxynivalenol (DON), fumonisin (B1 and B2) so-called $\mathrm{F}(\mathrm{B} 1, \mathrm{~B} 2)$, OTA, patulin (PAT), total aflatoxin (B1, B2, G1, and G2) (TA), and zearalenone (ZEN) - at the distribution stage, while data for domestic raw materials were collected from MAFRA which monitored three mycotoxins-DON, F(B1, B2), and $\mathrm{ZEN}$ - at the production stage based on a national monitoring program. Because each governmental authority provided different code system for their own dataset, the integrated database was conducted by standardizing different codes and compiling different datasets.

\section{Impact assessment processes}

The stepwise processes were designed to conduct impact assessment from macroscopic to microscopic (Fig. 1). Macroscopically, vulnerable mycotoxins due to climate change were determined by connecting the optimal growth temperature and humidity of for the origin fungus of target mycotoxins with changing patterns of the same meteorological variables. For the vulnerable mycotoxins, vulnerable raw materials were prioritized and change in vulnerable regions for the vulnerable raw materials was predicted sequentially.

\section{Prioritization of vulnerable mycotoxins due to climate change (Step 1)}

To identify environmental characteristics related to climate change, the optimal growth conditions were investigated for the origin fungus of target mycotoxins through MFDS reports [21] preliminary. After investigation, sequential processes were conducted to prioritize the vulnerability of target mycotoxins. At first, datasets for climate change 
Table 1 Information for internal datasets

\begin{tabular}{llll}
\hline Dataset & Description & No. of records & References \\
\hline Climate change & Past observation data (1999-2015) & 486,807 & {$[16]$} \\
& Future projected data (2015-2100) & $2,460,444$ & \\
Cultivation region & Korean cultivation area & 267,133 & {$[17]$} \\
Recipes for processed food & Processed food & 758 & {$[18]$} \\
Mycotoxin concentration in food & Processed food (distribution stage) & 61,229 & {$[19]$} \\
& Raw material (production stage) & 6063 & {$[20]$} \\
Total & & $3,281,676$ & \\
\hline
\end{tabular}

\section{Macroscopic assessment}

Fig. 1 Stepwise processes
implemented for impact assessment of mycotoxins due to climate change

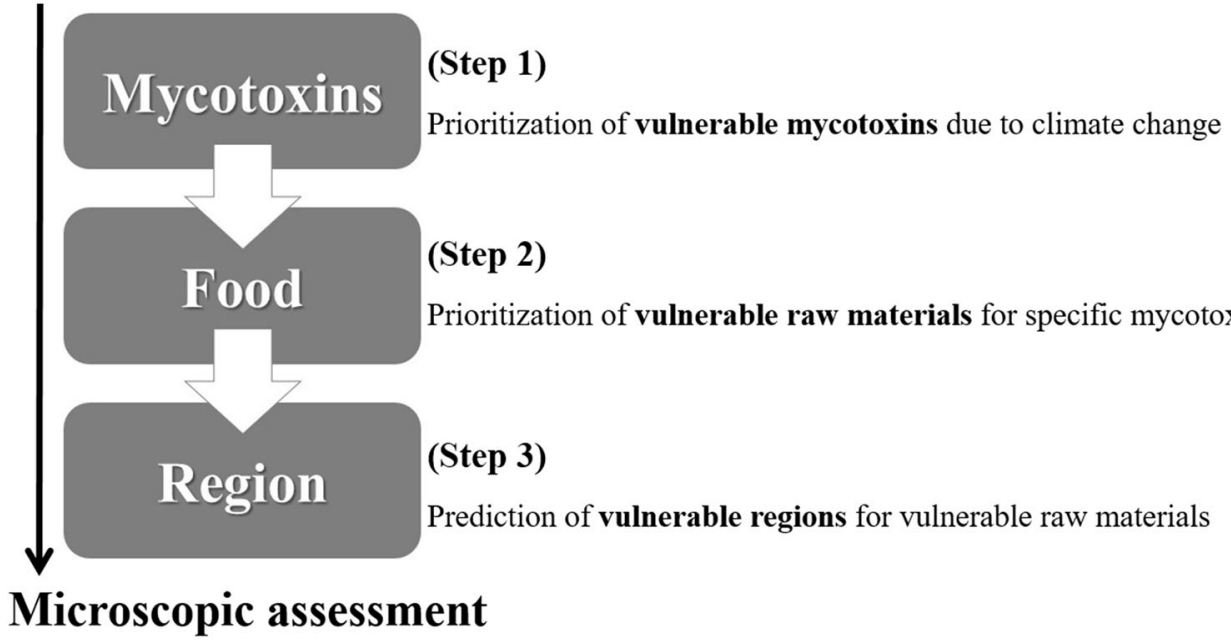

were divided into the past (1999-2015) and the future (2015-2100) and the days corresponding to the optimal growth conditions were calculated and connected. Based on future climate data, the trend line analysis was conducted for each RCP scenario with logarithmic linear regression models in a time-series manner. The statistical method used for prediction was the following:

$A d_{m, y_{i}}=\beta \log \left(y_{i}\right)+\alpha$

In Eq. 1, active days $\left(A d_{m, y_{i}}\right)$ for annual variables $\left(y_{i}\right)$ of target mycotoxins $(m)$ the slope $\beta$ and the intercept $\alpha$ were estimated, while log scale was applied to the $x$-axis due to the large scale of annual variable. Vulnerable mycotoxins were prioritized by positive correlation and high value order of $\beta$.

\section{Prioritization of vulnerable raw materials for specific mycotoxins (Step 2)}

This step was designed to identify the relationship between foods and mycotoxins for prioritization of vulnerable raw materials for specific mycotoxins determined in the Step 1.

Monitoring datasets for processed foods and raw materials were used to calculate the mean contamination level of specific mycotoxins. Recipe datasets for certain processed foods were also used to identify specific raw materials which corresponded to certain processed foods (contamination level $\geq 0$ ).

$V_{f, m}=\bar{c} l_{m, f} \times \mathrm{ca}_{\mathrm{f}}$

By using Eq. 2, vulnerability $(V)$ of food $(f)$ for mycotoxins $(m)$ was predicted by multiplying mean contamination level ( $\bar{c} l$ by cultivation area (ca). Vulnerable raw materials were prioritized by intensity order of $V$.

\section{Prediction of vulnerable regions for vulnerable raw materials (Step 3)}

This step was designed to identify risk and temporal change in vulnerable regions for vulnerable raw materials identified in Step 2.

$V_{r, f, m, y_{i}}=A d_{r, m, y i} \times \mathrm{ca}_{f, y_{i}}$

By using Eq. 3, annual $\left(y_{i}\right)$ vulnerability $(V)$ of region $(r)$ for food $(f)$ vulnerable to mycotoxins $(m)$ was predicted. Future temporal change in regional vulnerability was projected by multiplying the active days $(A d)$ of specific mycotoxins according to region based on past observation 
weather by cultivation area (ca) for food on an annual basis RCP data additionally.

\section{Environment for impact assessment}

To implement the primary database for overall impact assessment, Windows 2008 server was used in conjunction with the Oracle $10 \mathrm{~g}$ for relational database management system (RDBMS). For statistical analysis in a time-series manner, polynomial regression was utilized and applied to the system as a form of R-script with $\mathrm{R}$ version 3.3.1.

\section{Results}

\section{Prioritization of vulnerable target mycotoxins associated with climate change (Step 1)}

Table 2 shows the optimal growth conditions for fungus of target mycotoxins, which were applied as reference values. The mean optimal temperature and humidity ranged from 15 to $29.5{ }^{\circ} \mathrm{C}$ and from 88 to $93 \%$, respectively.

In time-series analysis by applying the prediction model (Eq. 1), current climate data (from 1999 to 2015) showed an increasing trend for every mycotoxin, while future climate data according to each RCP scenario indicated the maintained or decreased active days for four mycotoxins (DON, F (B1 and B2), PAT, and ZEN) and the increased active days for OTA and TA (Fig. 2).

Therefore, OTA and TA were prioritized as specific mycotoxins with high potential risks due to climate change in Korea. The sensitive analysis for temperature and humidity parameters was conducted to find relatively significant parameter for active days through applying the prediction model (Eq. 1) by using only one specific parameter.

For OTA, estimated slope for active days ranged from 0.1 to 0.7 in change of only temperature condition while ranged from 2.58 to 8.77 in change of only humidity condition. For TA, estimated slope for active days ranged from 0.1 to 0.7 in change of only temperature condition while ranged from 1.94 to 10.73 in change of only humidity condition. These results indicated that TA and OTA were highly sensitive for humidity.

From the standpoint of the RCP scenarios, the highest slopes of active days were demonstrated in RCP 8.5 scenarios for OTA and TA (Fig. 2). For TA, the estimated slopes increased in the order of RCP 2.6 (890.59), RCP 4.5 (1723.25), RCP 6.0 (2200.66) and RCP 8.5 (4605.60). OTA showed the increased patterns of active days but the estimated slope in RCP 6.0 (973.84) was lower than that in RCP 4.5 (1749.85). For other mycotoxins except OTA and TA, significant impact according to RCP scenarios was not found in these modeling results.

\section{Prioritization of vulnerable raw materials for specific mycotoxins (Step 2)}

Among 153 items including processed foods and raw materials, 79 and 77 items had contamination level for OTA and TA, respectively. By using recipe dataset, specific raw materials were mapped as surrogate material for processed food (contamination level $\geq 0$ ). By applying the prediction model (Eq. 2), vulnerability of all raw materials through multiplication of their cultivation area and mean contamination level for OTA and TA, was calculated respectively. Top 4 raw materials (chestnut, dried red pepper, perilla seed, and soy bean) with high vulnerability were identified as vulnerable raw materials for OTA and TA (Fig. 3).

\section{Prediction of vulnerable regions for vulnerable raw materials (Step 3)}

By applying the prediction model (Eq. 3), annual vulnerability, namely risk by regions for vulnerable raw materials identified in the Step 2, was calculated and the change in regional risk was predicted according to RCP scenarios from present (2017) to future (2100). Southwestern regions were main vulnerable regions in the present in Korea, while these regions except for chestnut would be moved to the north according to the climate change based on RCP scenarios (Fig. 4). As a result, increase in potential risk of northward regions was predicted for 4 raw materials (chestnut, dried red pepper, perilla seed, and soy bean) with vulnerability for
Table 2 The optimal active condition of origin fungi for target mycotoxins

\begin{tabular}{llclc}
\hline Mycotoxin & Origin fungi & Temperature & Relative humidity & Reference \\
\hline DON & Fusariums & $20-25$ & $97-99$ & {$[21]$} \\
F $\left(B_{1}, B_{2}\right)$ & Fusariums & $20-25$ & $85-97$ & \\
OTA & Penicilliums & $4-37$ & $80-85$ & \\
PAT & Penicilliums & $5-25$ & $95-98$ & \\
TA & Aspergillus & $28-35$ & $80-85$ & \\
ZEN & Fusariums & $15-30$ & $95-97$ &
\end{tabular}




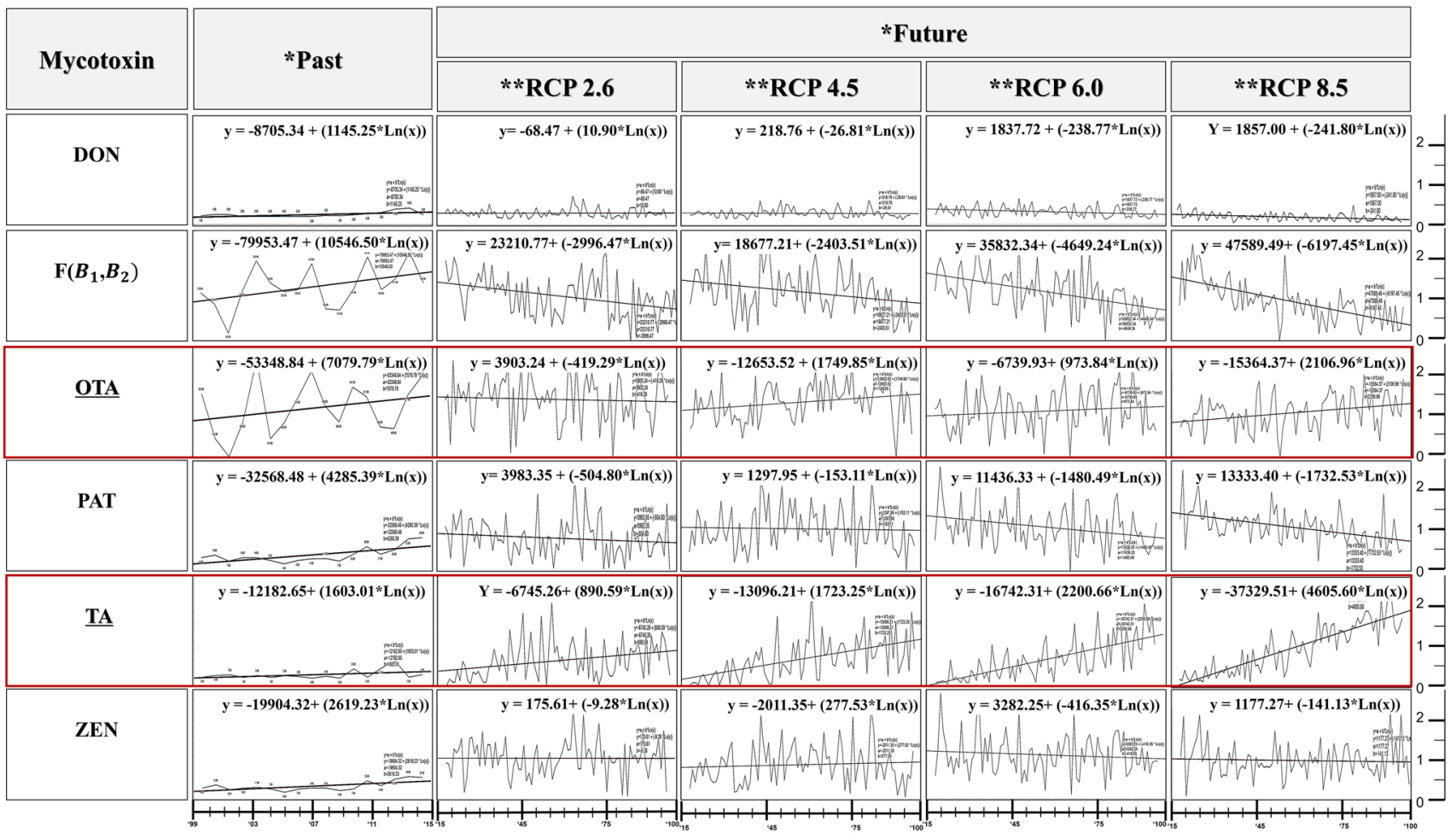

*Present $-X$ axis : 1999 2015 (year), Y axis : active days *100 (day)

$* * \mathbf{R C P}-\mathrm{X}$ axis : $2015 \sim 2100$ (year), $\mathrm{Y}$ axis : active days $* 100$ (day)

Fig. 2 The results of time-series analysis for target mycotoxins

specific mycotoxins (OTA and TA) due to climate change in Korea.

\section{Discussion}

To predict changes in mycotoxin risks due to climate change, prediction models were developed and implemented in this study.

Many studies [6-15] using prediction models for mycotoxin risks owing to climate change have been conducted. One study was conducted to predict contamination levels of DON in winter wheat using a prediction model developed with cultivation data collected from 2001 to 2008 in 425 regions in the Netherlands [6]. Similarly, a model was developed to estimate the impacts of climate change on contamination levels of DON for wheat cultivated in Northwestern Europe [7]. Datasets for wheat phenology and climate were used as input variables in this model, and wheat flowering date, period between flowering and full maturation, wheat resistance grade for Fusarium species (low/mid/ high), and diverse climate variables such as temperature, rainfall, and relative humidity were also included. In particular, climate data contained a total of 50 simulations per grid, with 851 grids of $50 \times 50 \mathrm{~cm}$ [8]. To identify climate change impacts, one study [9] developed a prediction model using meteorological data from eight meteorological stations in northern Italy collected over 6 years (2006-2011). Several variables and parameters were determined to predict (1) growth and development of Aspergillus carbonarius in grapes and (2) the increase in OTA in berries. As a result, intermediate variables were berries status and growth stage of grapes, while rate variables were dispersal rate, germination rate, growth rate, infection rate, and OT production rate, and parameters were air temperature, relative humidity, rainfall, and water activity.

In addition, prediction models were developed for mycotoxin risks associated with climate change [6-15] by using climate change data and investigating the optimal environments for mycotoxin growth through studies and reports from relevant organizations including governmental organizations. However, as pointed out in one study [10], precedent studies have limitations, for example, only considering mycotoxins such as DON, those produced by Fusarium species, and OTA or foods such as grapes, US maize, and winter wheat.

On the other hand, our study utilized four datasets including climate change from the atmospheric environment, Korean cultivation conditions, recipes, and concentrations of target six mycotoxins in processed foods to implement the prediction model In addition, our study targeted total six kinds of mycotoxins and overall foods 


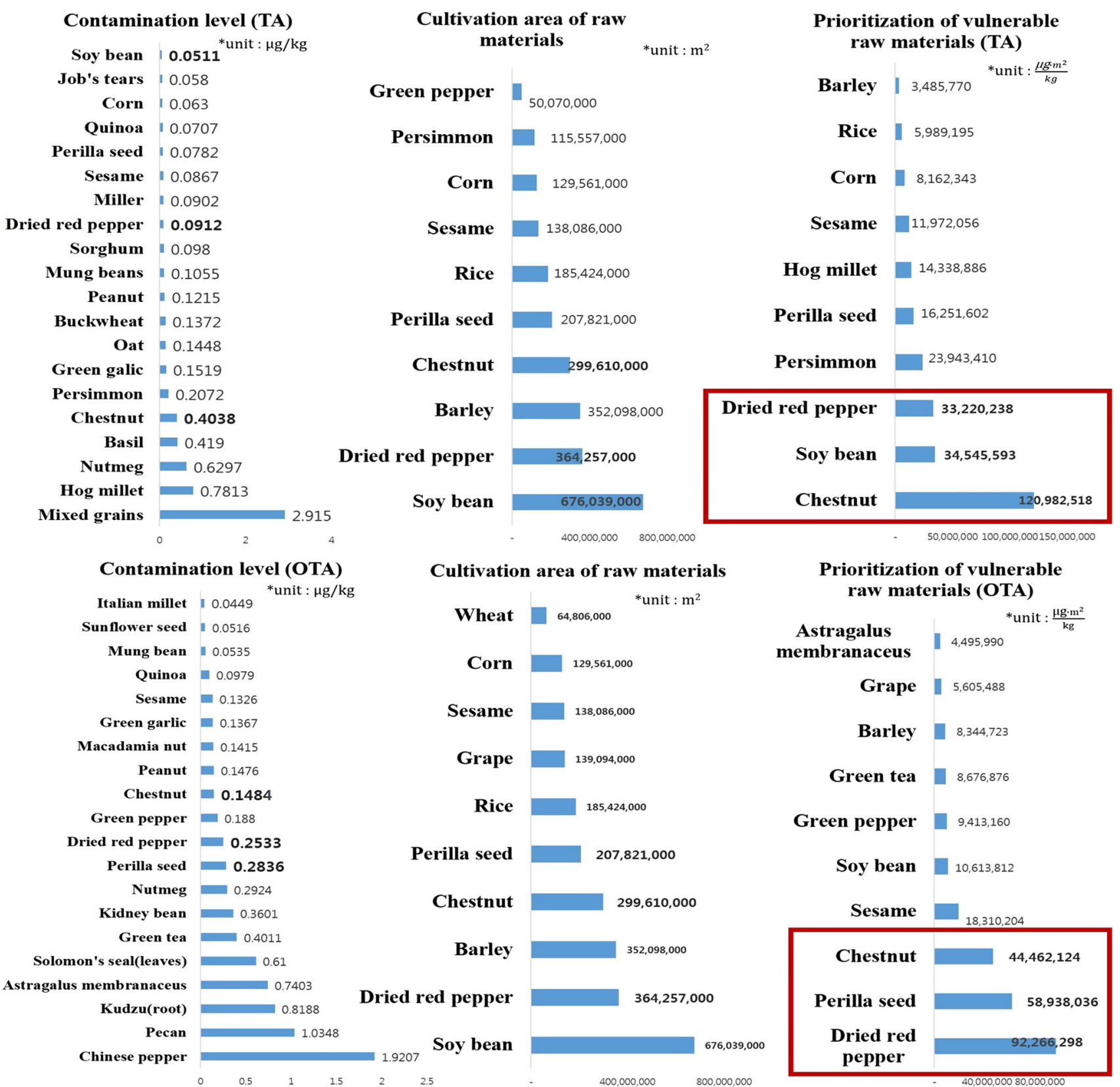

Fig. 3 Prioritized results for vulnerable raw materials for OTA and TA

cultivated in Korea. By using prediction models, the combination of vulnerable mycotoxins and raw materials was derived by considering cultivation area and contamination levels in the Korean peninsula, and then a vulnerability moving map from the present to 2100 was finally drawn. This combination of vulnerable mycotoxins and raw materials could be used as a marker for continuous monitoring tool of future mycotoxin changes for adaptation to climate change. In particular, this combination can be utilized for sampling optimization such as item types, the amounts of items, and the regions for monitoring in the progress of planning for long-term agricultural safety policies conducted by the nation.

In one study [8], two kinds of prediction models (Model 1 and 2) were developed with diverse data for region, spraying, wheat resistance level, and flowering date and various climatic variables related to rainfall, relative humidity, and temperature in different critical periods of wheat cultivation. Model 1, for farmers who cultivate wheat, predicted the contamination levels of DON during the wheat growing season and thereby supported pest management by using appropriate fungicides. Model 2, for industries and food safety authorities, predicted DON 
Fig. 4 Predicted results for vulnerable regions for OTA and TA of raw materials

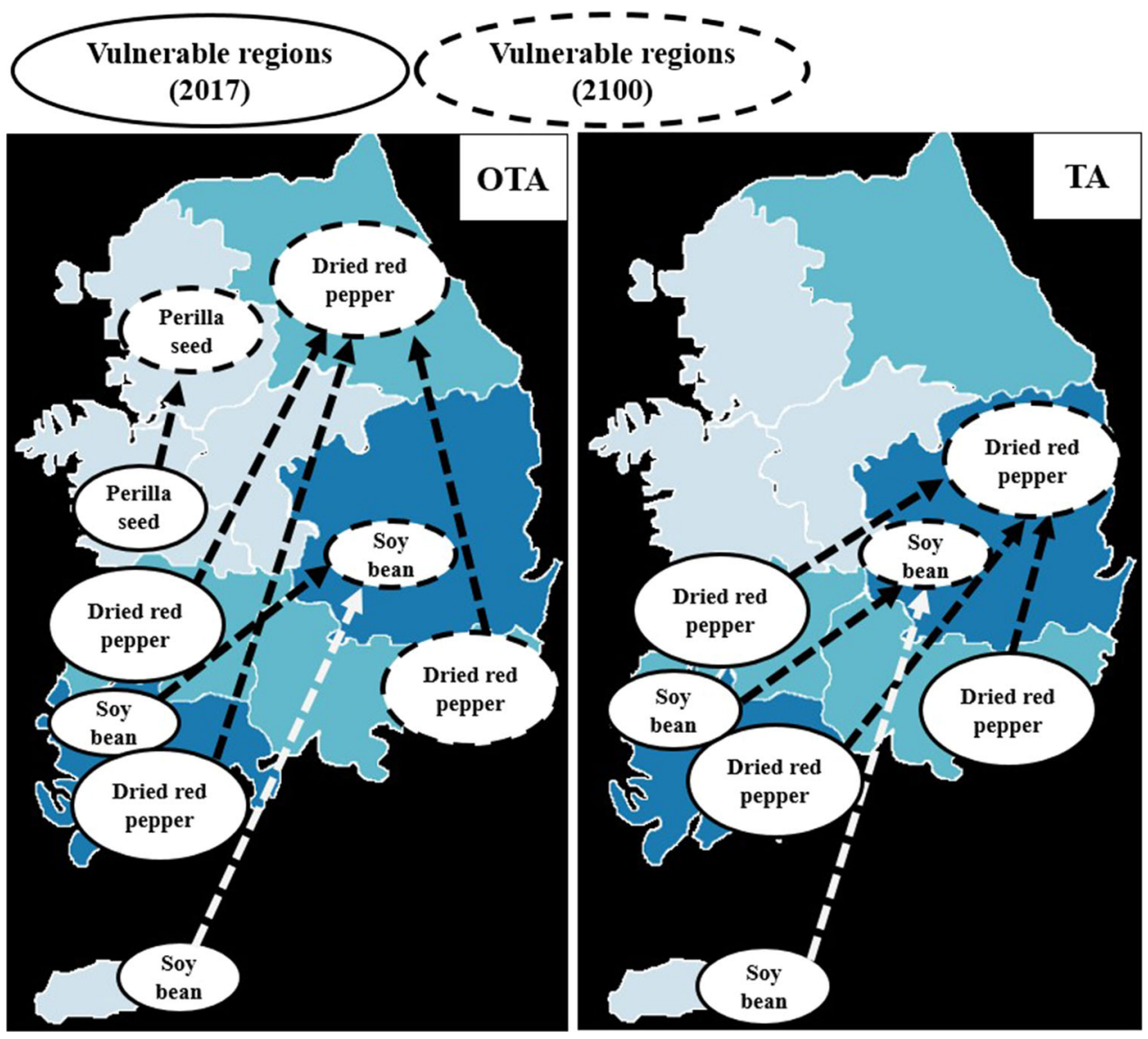

levels in the soil by considering the cultivation period of all wheat cultivated in the Netherlands [8].

However, our study did not utilize datasets such as wheat resistance level and flowering date and climate variables such as rainfall. In addition, our study was not conducted for specific target such as farmers. Therefore, to more accurately predict more mycotoxins that pose risks in conjunction with climate change than was accomplished using the present model, updated prediction model will be needed to include (1) cultivation area with pesticides contamination in Korea and the diversity of cultivated agricultural foods, (2) datasets for cultivation condition by crops such as cultivar resistance level, flowering date, and (3) diverse various climatic variables, such as in different critical periods of crops, and length between flowering and harvest. In addition, it is necessary to develop specialized prediction models targeting specific groups such as farmers, industries, and laboratories to provide customized prediction results by users.

In conclusion, this study predicted risks for mycotoxin due to climate change in Korea. The core datasets, such as climate change, contamination-level monitoring, recipes for processed food and cultivation area in specific regions, were collected and compiled for prediction preliminarily. In addition, three prediction models were conducted for vulnerable mycotoxins, materials and food by using this core datasets. Among six target mycotoxins (DON, F(B1,B2), OTA, PAT, TA, and ZEN), OTA and TA were identified as specific vulnerable mycotoxins. For OTA and TA, 4 raw materials (chestnut, dried red pepper, perilla seed, and soy bean) were derived as vulnerable raw materials and change in their vulnerable regions was predicted to be moved to the northward area in Korea. These results can be contributed to conduct risk management policy such as long-term national sampling plan for mycotoxins in food by considering climate change environment in Korea.

Acknowledgments This work was supported by the Ministry of food and drug safety, republic of Korea (Grant Number 17162MFDS066) in 2017.

\section{References}

1. Food Standards Agency (2015) Impacts of climate change on food safety. https://www.food.gov.uk/sites/default/files/impactof-climate-change.pdf. Accessed 10 April 2018

2. Magan N, Medina A, Aldred D (2011) Possible climate-change effects on mycotoxin contamination of food crops pre- and postharvest. Plant Pathol 60:150-163 
3. Prandini A, Sigolo S, Filippi L, Battilani P, Piva G (2009) Review of predictive models for Fusarium head blight and related mycotoxin contamination in wheat. Food Chem Toxicol 47:927-931

4. Paterson RRM, Lima N, Taniwaki MH (2014) Coffee, mycotoxins and climate change. Food Res Int 61:1-15

5. IPCC (2014) Climate change 2014 mitigation of climate change working group III contribution to the fifth assessment report of the intergovernmental panel on climate change. https://www.ipcc. ch/pdf/assessment-report/ar5/wg3/ipcc_wg3_ar5_frontmatter. pdf. Accessed 3 Jan 2017

6. Van der Fels-Klerx HJ, Burgers SL, Booij CJ (2010) Descriptive modeling to predict deoxynivalenol in winter wheat in the Netherlands. Food Addit Contam 27:636-643

7. Van der Fels-Klerx HJ, Goedhart PW, Elen O, Börjesson T, Hietaniemi V, Booij CJ (2012) Modeling deoxynivalenol contamination of wheat in northwestern Europe for climate change assessments. J Food Prot 75:1099-1106

8. Van der Fels-Klerx HJ, Liu C, Battilani P (2016) Modeling climate change impacts on mycotoxin contamination. World Mycotoxin J 9:717-726

9. Paola B, Marco CL (2015) OTA-grapes: a mechanistic model to predict ochratoxin A risk in grapes, a step beyond the systems approach. Toxins 7:3012-3029

10. Lobell DB, Burke MB, Tebaldi C, Mastrandrea MD, Falcon WP, Naylor RL (2008) Prioritizing climate change adaptation needs for food security in 2030. Science 319:607-610

11. Bindi M, Olesen JE (2011) The responses of agriculture in Europe to climate change. Reg Environ Change 11:151-158
12. Ewert F, Rounsevell M, Reginster I, Metzger M, Leemans R (2005) Future scenarios of European agricultural land use: I. Estimating changes in crop productivity. Agric Ecosyst Environ 107:101-116

13. Falloon P, Betts R (2010) Climate impacts on European agriculture and water management in the context of adaptation and mitigation - the importance of an integrated approach. Sci Total Environ 408:5667-5687

14. Olesen JE, Carter T, Diaz-Ambrona CH, Fronzek S, Heidmann T, Hickler T, Holt T, Minguez M, Morales P, Palutikof J (2007) Uncertainties in projected impacts of climate change on European agriculture and terrestrial ecosystems based on scenarios from regional climate models. Clim Change 81:123-143

15. EC (2009) Adapting to climate change: towards a European framework for action. http://tinyurl.com/hj7d9df. Accessed 3 Jan 2017

16. [Dataset] KMA, Present observation database (1999-2015) (2015)

17. AT/FIS (2016) Cultivation area of crops (2011-2014). http:// www.atfis.or.kr/indexP.html Accessed 10 April 2017

18. [Dataset] MFDS (2016) Recipes for processed food (2008-2012)

19. [Dataset] MFDS (2016) Concentration database of mycotoxins in foods (processed food) (2012-2015)

20. Lee JK (2012) The study on safety control of National Pesticide Residues for Foods. Report no. 09072MFDS997. https://rnd. mfds.go.kr/\#!RDCAC09F01View. Accessed 10 April 2018

21. MFDS (2007) Mycotoxins in food. http://theme.archives.go.kr/ viewer/common/archWebViewer.do?bsid=200700199666\&gubun= search. Accessed 5 May 2018 